\title{
Electron Singularities, Matter Wave Catastrophes, and Vortex Lattice Singularimetry
}

\author{
T. C. Petersen ${ }^{1}$, M. Weyland ${ }^{2,3}$, D. M. Paganin ${ }^{1}$, T. P. Simula ${ }^{1}$, S. A. Eastwood ${ }^{1}$, A. I. Bishop ${ }^{1}$ and M. \\ J. Morgan ${ }^{1}$ \\ 1. School of Physics and Astronomy, Monash University, Clayton, Australia \\ 2. Department of Materials Engineering, Monash University, Clayton, Australia \\ 3. Monash Centre for Electron Microscopy, Monash University, Clayton, Australia
}

Singularities in wave fields, such as phase vortices, are of substantial interest for light optics, matter waves and x-ray optics. New theoretical insights [1] and cutting edge nanofabrication techniques have recently created a wide variety of electron vortex beams [2, 3] for the transmission electron microscope (TEM), which can provide spectroscopic information pertaining to the magnetic properties of nanoscale specimens [2]. The rich diffraction physics of singular optics is vast and many concepts can be directly translated to electron microscopy. Interesting physics has been discovered by highlighting differences between electron and optical vortex beams, such as a remarkable interplay between Zeeman coupling, Landau levels and Gouy phases in the TEM [4] or the Faraday effect for electron vortex beams propagating in a magnetic field, which interestingly occurs in vacuum [5].

Our singular electron optics experiments began by considering ways in which to spontaneously nucleate vortices in the TEM using aberrations. Using insights from Berry et al. [6], we have created electron diffraction catastrophes [7] to produce distorted lattices of vortices by imposing probe forming aberrations in a $\operatorname{Titan}^{3}$ 80-300 TEM (FEI), which can correct both the illumination and imaging lenses. More recently, we used electron aberrations to produce the elliptic umbilic catastrophe and have measured the non-trivial three dimensional (3D) architecture of the hyperbolic umbilic.

The diffraction catastrophe experiments naturally led to measurements of the Gouy phase anomaly [8, 9] for astigmatic electron waves [10] using in-line holography. The Gouy anomaly describes quantized phase changes incurred by rays on the optic axis, which pass through a caustic. By examining the 3D form of the astigmatic caustic in the TEM, we sought to derive an intuitive explanation of the Gouy phase anomaly for focused electron waves, based upon the probability density in the vicinity of line foci. By considering quantum fluctuations near the optic axis, we united several disparate theoretical interpretations within the framework of focussed paraxial electron waves [11]. Specifically, we related the anomaly to Heisenberg momentum fluctuations, statistical confinement, Berry's geometric phase, and Keller's quantized semi-classical phase changes, known as Maslov indices. We predicted direct encoding of Gouy phases in Gabor holograms, which could be integrated using quantum 'weak measurements' of the local momentum. Preliminary data demonstrates this approach, as in shown Figure 2, which is more accurate than our original in-line holography results.

Our studies of electron caustics informed new approaches for creating vortices in Bose Einstein Condensates (BEC) using quantum gas analogous of aberrated focusing, based upon external trapping potentials, which were confirmed using simulations of the Gross-Pitaevskii equation, including nonlinear interactions [12]. The BEC study created new insights for interpreting recent Bose nova experiments and the detection of dipole-dipole interactions. 
We are currently motivated by practical applications of vortex lattices for interferometry [13], which we have been developing using light optics. We have derived a quantitative form of 'singularimetry', in which a 3-beam lattice of optical vortices provides an algebraic and localized method for measuring phase shifts imparted by an absorbing and refracting specimen in one arm of a 3-beam interferometer. For certain microscopy techniques, it may not be technologically feasible to use multiple beam splitters in this manner. To circumvent this issue, we have developed an alternative differential form of singularimetry, which utilizes vortices and gradient singularities as topological fiducial markers in a structured illumination context. This new approach provides a concise analytic measurement of phase gradients imparted by refracting specimens, yielding quantitative information that is both local and deterministic. We have accurately quantified our phase gradient experiments to demonstrate that lattices of wave field singularities can be used to detect subtle specimen variations with high precision.

[1] KY Bliokh, YP Bliokh, S Savel'ev and F Nori, Phys. Rev. Lett. 99 (2007), p. 190404.

[2] J Verbeeck, H Tian and P Schattschneider, Nature 467 (2010), p. 301.

[3] BJ McMorran et al., Science 331 (2011) p. 192.

[4] KY Bliokh, P Schattschneider, J Verbeeck and F Nori, Phys. Rev. X 2 (2012), p. 041011.

[5] C Greenshields, RL Stamps and S Franke-Arnold, New J. Phys. 14 (2012), p. 103040.

[6] MV Berry, JF Nye and FJ Wright, Phil. Trans. A 291 (1979), p.1382.

[7] TC Petersen et al., Phys. Rev. Lett. 110 (2013) p. 033901

[8] TD Visser and E Wolf, Opt. Commun. 283 (2010), p. 3371.

[9] G Guzzinati, P Schattschneider, KY Bliokh, F Nori and J Verbeeck, Phys. Rev. Lett. 110 (2013), p. 093601.

[10] TC Petersen et al. Phys. Rev. A 88 (2013) p. 043803.

[11] TC Petersen et al. Phys. Rev. A 89 (2014) p. 063801.

[12] TP Simula, TC Petersen and DM Paganin, Phys. Rev. A 88 (2013), p. 043626.

[13] SA Eastwood, AI Bishop, TC Petersen, DM Paganin and MJ Morgan, Opt. Express 20 (2012) p. 13947.

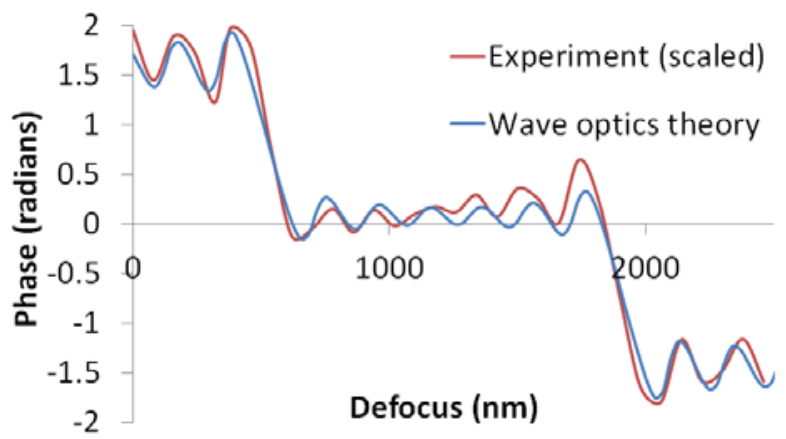

Figure 1. Gouy phase jumps measured directly from an astigmatic electron caustic. The experiment has been vertically scaled to match theory, heuristically accounting for partial coherence and image blur.
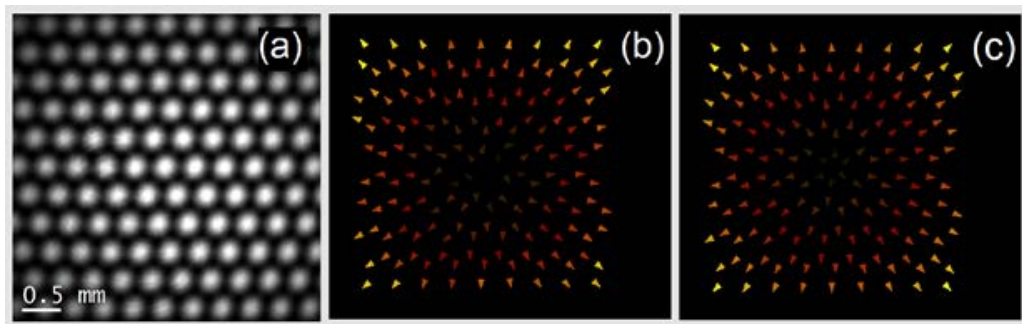

Figure 2. a) Raw intensity of a three beam vortex lattice, b) experimental phase gradients measured at vortex locations c) theoretical vector field at vortex locations; colors indicate gradient magnitude. 